\title{
Pharmacodynamic modeling of adverse effects of anti-cancer drug treatment
}

\author{
A. H. M. de Vries Schultink ${ }^{1}$ - A. A. Suleiman ${ }^{2}$ - J. H. M. Schellens ${ }^{3,4}$ • J. H. Beijnen ${ }^{1,4}$. \\ A. D. R. Huitema ${ }^{1}$
}

Received: 8 December 2015 / Accepted: 16 February 2016 /Published online: 26 February 2016

(C) The Author(s) 2016. This article is published with open access at Springerlink.com

\begin{abstract}
Purpose Adverse effects related to anti-cancer drug treatment influence patient's quality of life, have an impact on the realized dosing regimen, and can hamper response to treatment. Quantitative models that relate drug exposure to the dynamics of adverse effects have been developed and proven to be very instrumental to optimize dosing schedules. The aims of this review were (i) to provide a perspective of how adverse effects of anti-cancer drugs are modeled and (ii) to report several model structures of adverse effect models that describe relationships between drug concentrations and toxicities. Methods Various quantitative pharmacodynamic models that model adverse effects of anti-cancer drug treatment were reviewed.

Results Quantitative models describing relationships between drug exposure and myelosuppression, cardiotoxicity, and graded adverse effects like fatigue, hand-foot syndrome
\end{abstract}

A. H. M. de Vries Schultink ah.d.vriesschultink@nki.nl

1 Department of Pharmacy and Pharmacology, Antoni van Leeuwenhoek-The Netherlands Cancer Institute and MC Slotervaart, Louwesweg 6, 1066 EC Amsterdam, The Netherlands

2 Department of Pharmacology, Clinical Pharmacology Unit, University Hospital of Cologne, Gleueler Str. 24, 50931 Cologne, Germany

3 Department of Clinical Pharmacology, Antoni van LeeuwenhoekThe Netherlands Cancer Institute, Plesmanlaan 121, 1066 CX Amsterdam, The Netherlands

4 Science Faculty, Utrecht Institute for Pharmaceutical Sciences (UIPS), Division of Pharmacoepidemiology and Clinical Pharmacology, Utrecht University, P.O. Box 80082, 3508 TB Utrecht, The Netherlands
(HFS), rash, and diarrhea have been presented for different anti-cancer agents, including their clinical applicability.

Conclusions Mathematical modeling of adverse effects proved to be a helpful tool to improve clinical management and support decision-making (especially in establishment of the optimal dosing regimen) in drug development. The reported models can be used as templates for modeling a variety of anti-cancer-induced adverse effects to further optimize therapy.

Keywords Modeling $\cdot$ Pharmacodynamics $\cdot$ Adverse effects $\cdot$ Anti-cancer drug treatment

\section{Introduction}

Adverse effects are a major problem in the treatment with both cytotoxic drugs and newer targeted therapies, resulting in dose reductions, dose delays, and treatment cessation. Toxicity can impair quality of life, jeopardize treatment adherence, and necessitate dose reductions and dose delays, which can negatively affect response to treatment and outcome [1,2]. The tendency in cytotoxic anti-cancer drug treatment is to dose drugs around the maximum tolerated dose (MTD), which assumes that the highest possible dose achieves the maximum effect [3]. Adverse effects are, therefore, frequently observed during treatment with cytotoxic drugs. Targeted therapies are expected to have less toxicity, mainly because of two reasons: (i) these therapies are specific to a tumor target and induce less off target toxicity and (ii) targeted therapies might have maximum target inhibition at lower concentrations than the MTD. The latter has led to the suggestion that targeted therapies should be dosed around the optimal biological dose (where target saturation is maximal) rather than the MTD. However, definition of the optimal biological dose is hampered by the lack of validated biomarkers for efficacy, lack of information on the relation between 
target binding and survival measures, and yet information on the highest possible dose remains of value [4]. As a consequence, targeted therapies are still often dosed around the MTD [2].

Adverse effects related to cancer therapy are typically graded by the National Cancer Institute's Common Terminology Criteria for Adverse Events (NCI-CTC-AE). A conventional and common approach to analyze toxicity data is to calculate the proportion of patients that experienced a certain (severe) grade of toxicity [5]. Subsequently, these proportions can be statistically related to dosing groups, area under the plasma concentration-time curve (AUC) or other summary variables for exposure [6]. However, it is essential to have information on the dynamic relation between exposure and toxicity, which provides information on when the toxicity occurs, what the severity is over time, and if or when the adverse effect is reversed. For this purpose, quantitative models are becoming increasingly important. These models describe the time course of toxicities related to exposure, as will be described throughout this review.

Since modeling adverse effects is becoming increasingly important in anti-cancer drug treatment and drug development, an overview of existing modeling approaches can be helpful for future research. Therefore, the aim of this review is to give a perspective of modeling adverse effects of anti-cancer drugs and report several model structures that describe relationships between drug concentrations and toxicities, thereby focusing on the fixed effects of non-linear mixed effects models.

\section{Modeling adverse effects}

\section{Myelosuppression}

Myelosuppression is the leading dose-limiting toxicity in treatment with cytotoxic agents. Hematological toxicity consists of low leukocyte, thrombocyte, and platelet counts, potentially leading to life-threatening infections, anemia, and bleeding. Neutropenia, a subtype of leucopenia, is the most common and serious hematologic toxicity observed during treatment with cytotoxic anti-cancer drugs [7]. Myelosuppression can necessitate dose reductions and dose delays, potentially resulting in suboptimal drug exposure. Early approaches to describe hematological toxicity aimed at finding correlations between summary variables of exposure and summary variables of myelosuppression, not taking into account the complete time course of either drug concentration or myelosuppression. Survival fraction of blood cells or percentage change in blood count were typically used as summary variables for myelosuppression, whereas average drug concentration, AUC, or peak drug concentration were used to summarize exposure [8-10]. These models have major limitations such as poor predictive value and lack of description of the dynamics of toxicity.

\section{Empirical models}

The first models describing the complete time course of myelosuppression were empirical models. One model described the time course of leucopenia in patients treated with etoposide and used a lag time to account for the delay in the myelosuppressive effect and a cubic spline function, which represented the deviation of white blood cell (WBC) count from baseline [11]. An $E_{\max }$ model described the decline in WBC count from baseline, which was dependent on the effective concentration of etoposide. A similar empirical model was published for paclitaxel-induced leucopenia [12].

\section{Semi-mechanistic models}

Currently, a more mechanistic modeling approach is used. Mechanistic models mimick the physiological processes of hematopoiesis. Generally, this improves the predictive value of the model, since the mechanism-related parameters represent actual physiological processes. Hematopoiesis is characterized by proliferation of progenitor cells in the bone marrow, followed by maturation and degradation of blood cells [13]. To make useful models for pharmacokinetic and pharmacodynamic (PK-PD) analysis, several simplified semi-mechanistic models have been developed (Table 1) [14-19]. These semimechanistic models are all characterized by a proliferation cell compartment or progenitor compartment containing cells that have self-renewing capacity and a compartment representing circulating cells. In order to account for the maturation process, that delays the effect of the drug, either lag time or oneto multiple-transit compartments are added to the model structure. In some of the semi-mechanistic models, a feedback loop is incorporated to describe the rebound of blood cells, exceeding the blood count at baseline, which occurs when drug concentrations decrease. Typically, this feedback effect is driven by the amount of circulating blood cells, which affects the rate of proliferation in the progenitor compartment. Drug effects were modeled to affect the proliferation rate or the amount of progenitor cells. Model characteristics of five published semimechanistic models are summarized in Table 1. A general model structure for myelosuppression is depicted in Fig. 1.

The first semi-mechanistic model developed used a twocompartment indirect response model to describe the time course of leucopenia in paclitaxel- and etoposide-treated patients [14]. The drug inhibited the proliferating cells only during a sensitive stage. This model is the only model that used lag time to mimick the maturation process instead of using transit compartments.

In 2000, Friberg et al. published a semi-mechanistic model, modeling the absolute neutrophil counts (ANCs) in 2'-deoxy2'-methylidenecytidine (DMDC)-treated patients [15]. This model contained three additional proliferating compartments and five non-mitotic compartments. The first-order 
Table 1 Semi-mechanistic models describing blood count over time

\begin{tabular}{|c|c|c|c|c|c|c|c|}
\hline \multicolumn{2}{|l|}{ Reference } & \multirow{2}{*}{$\begin{array}{l}\text { Drug } \\
\text { Paclitaxel }\end{array}$} & \multirow{2}{*}{$\begin{array}{l}\text { Observed variable } \\
\text { WBC }\end{array}$} & \multirow{2}{*}{$\frac{\operatorname{Par}^{\mathrm{a}}}{4}$} & \multirow{2}{*}{$\frac{\operatorname{Tr}^{\mathrm{b}}}{\text { Lag time }}$} & \multirow{2}{*}{$\frac{\mathrm{k}_{\text {prol }}{ }^{\mathrm{c}}}{\text { Zero order }}$} & \multirow{2}{*}{$\frac{\text { Drug effect }}{E_{\max }}$} \\
\hline Minami (1998) & [14] & & & & & & \\
\hline Friberg (2000) & {$[15]$} & DMDC & ANC & 7 & 9 & Zero order & $E_{\max }$ \\
\hline Zamboni (2001) & {$[16]$} & Topotecan & ANC & 4 & 1 & Zero order & $E_{\max }$ \\
\hline Friberg (2002) & [17] & Docetaxel, etoposide, and paclitaxel & ANC WBC & 5 & 3 & First order & Linear \\
\hline Panetta (2003) & [18] & TMZ & ANC & 5 & 2 & First order & $E_{\max }$ \\
\hline Bulitta (2009) & [19] & Paclitaxel paclitaxel EL & ANC & 5 & $1^{\mathrm{d}}$ & Zero order & Linear \\
\hline
\end{tabular}

$W B C$ white blood cell count, $A N C$ absolute neutrophil count, $T M Z$ temozolomide

${ }^{\text {a }}$ Number of parameters estimated in pharmacodynamic model

${ }^{b}$ Number of transit compartments or if lag time is used

${ }^{\mathrm{c}}$ Proliferation rate constant

${ }^{\mathrm{d}}$ Maturating pool of cells

elimination from the first progenitor compartment was proportional to the DMDC concentration. A fraction of the effect of DMDC on the first progenitor compartment was added to the other proliferating compartments. The non-mitotic compartments were not affected by DMDC concentration. Cytotoxic anti-cancer drugs only affect proliferating cells; therefore, this is a more elegant way of incorporating the maturation chain and the delay in drug effect as compared to using lag time.

The most well-known semi-mechanistic model for myelosuppression by Friberg et al. published in 2002 is often referred to as the golden standard for modeling the time course of myelosuppression [17]. Development was based on data from docetaxel-, etoposide-, and paclitaxel-treated patients, in whom ANC and WBC were measured. The model structure is described by the following equations:

$$
\begin{aligned}
& \frac{d \text { Prol }}{d t}=k_{\text {prol }} \cdot P_{\text {cells }} \cdot\left(1-E_{d r u g}\right) \cdot\left(\frac{\text { Circ }_{0}}{\text { Cir }}\right)^{\gamma}-k_{t r} \cdot P_{\text {cells }} \\
& \frac{\mathrm{dTransit} 1}{d t}=k_{t r} \cdot P_{\text {cells }}-k_{t r} \cdot \text { Transit1 } \\
& \frac{\text { dTransit2 }}{d t}=k_{t r} \cdot \text { Transit } 1-k_{t r} \cdot \text { Transit2 }
\end{aligned}
$$

$$
\begin{aligned}
& \frac{\mathrm{dTransit3}}{d t}=k_{t r} \cdot \text { Transit2- } k_{t r} \cdot \text { Transit3 } \\
& \frac{\mathrm{dCirc}}{d t}=k_{t r} \cdot \text { Transit3 }-k_{\mathrm{circ}} \cdot \text { Circ }
\end{aligned}
$$

where $k_{\text {prol }}$ represents the first-order proliferation input, $P_{\text {cells }}$ represents the amount of cells in the proliferation compartment, and $E_{\text {drug }}$ represents the drug effect. The feedback mechanism was described by $\left(\mathrm{Circ}_{0} \mathrm{Circ}\right)^{\gamma}$, where $\mathrm{Circ}_{0}$ is the ANC or WBC blood count at baseline, Circ is the amount of circulating blood cells, and $\gamma$ is the parameter estimate to determine the impact of the feedback. Transits 1-3 represent the amount of cells in the transit compartments, and $k_{\text {tr }}$ represents the rate constant between compartments. Degradation of circulating cells is described by the rate constant $k_{\text {circ }}$. The first-order proliferation input is different from previously described models, which used a zero-order rate constant of proliferation. It was assumed that the proliferation, maturation, and degradation rate constants were equal. Therefore, only three system-related parameters were estimated. An analysis was conducted to evaluate the consistency of the systemrelated parameters, by fixing them and re-estimating the drug-related parameters. The drug-related parameter estimates were comparable. Additionally, the system-related parameter

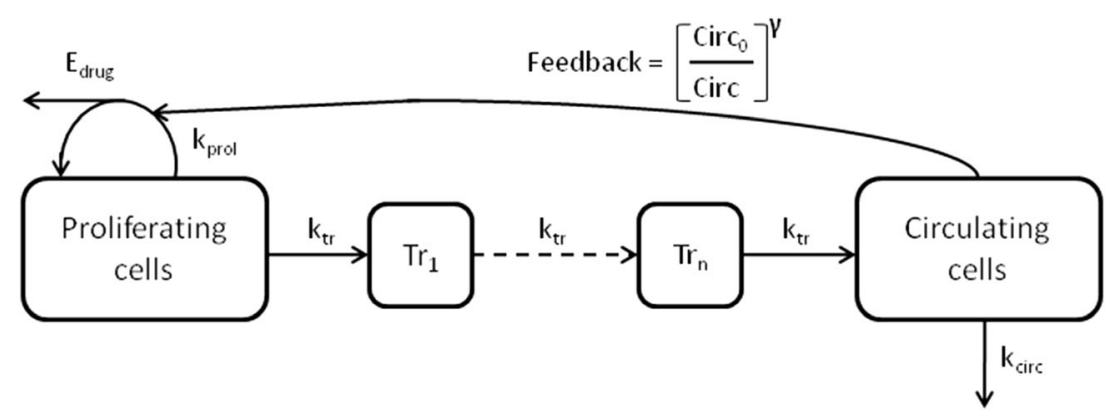

Fig 1 General model structure for myelosuppression. $E_{d r u g}$ drug effect, $k_{t r}$ maturation rate constant, $k_{\text {prol }}$ proliferation rate constant, $k_{c i r c}$ degredation rate constant, $T_{n}$ transition compartment, $\operatorname{Circ}_{0}$ circulating cells at baseline, Circ amount of circulating cells, and $\gamma$ factor for impact of feedback 
estimates were similar for different drugs, enabling interchangeability of the model between drugs. In 2003, a model with similar characteristics was published [18].

The most recent model for myelosuppression is a multiple-pool life span model for neutropenia [19]. This model estimates the life span of cells staying in a certain stage, starting with duration of the cells in the progenitor compartment, followed by duration in the maturation compartment, and lastly by duration in the circulation until degradation of the neutrophils. The model requires extensive computing with use of 17 differential equations.

In conclusion, the model published by Friberg et al. in 2002 is most frequently used and has several advantages above the other models [17]. This model has a clear separation between drug-related and systemrelated parameters, making the model applicable to different drugs. Additionally, the model only estimates few system-related parameters, allowing it to model sparse data sets. Four of the reported models in this review have been compared to the model by Friberg et al. using ANC data from patients treated with a Plk-1 inhibitor [20]. The results of this analysis implicated that none of the models showed superior performance to the model by Friberg et al. [17, 20]. Lastly, this model has been used in multiple studies with different drugs and research aims and has also been modified and applied to describe thrombocytopenia in patients treated with cytotoxic anti-cancer drugs or targeted therapies [21-33]. The extensive application, the limited number of system-related parameters, and the overall experience with this model make it the best starting point for modeling myelosuppression.

\section{Cardiovascular adverse effects}

For both cytotoxic and targeted therapies, cardiovascular toxicity has been reported. Anthracyclines can cause arrhythmias during or after administration and chronic cardiac toxicity, resulting in irreversible left ventricular dysfunction and congestive heart failure (CHF) [34]. Anthracycline-induced $\mathrm{CHF}$ has been related to dose, where patients receiving a cumulative dose of $550 \mathrm{mg} /$ $\mathrm{m}^{2}$ doxorubicin were at increased risk of developing CHF [35]. Trastuzumab has also been associated with cardiac complications, inducing (reversible) left ventricular systolic dysfunction, which can result in CHF [36]. Additionally, tyrosine kinase inhibitors, targeting the vascular-endothelial growth factor receptor (VEGFR), have been associated with hypertension and cardiac arrhythmias as well as other systemic anti-cancer drugs [37-39].

\section{Hypertension}

Pharmacodynamic models have been developed for lenvatinib- and sunitinib-induced hypertension, describing the change in blood pressure (BP) over time in relation to treatment (Table 2) [40, 41]. The relationship between lenvatinib exposure and increase of diastolic (d) and systolic (s) BP was best described by an indirect response model with two effect models for $\mathrm{dBP}$ and sBP. The plasma concentration of lenvatinib at the time point of BP measurement was used as input rate for the indirect effect model with a linear function [40]. Additionally, this model included the effect of antihypertensive therapy on blood pressure. A similar indirect response model is used to describe the increase of $\mathrm{dBP}$ in sunitinib-treated patients [41].

\section{Cardiotoxicity}

Cardiotoxicity as expressed as decline in left ventricular ejection fraction (LVEF) was used to develop a pharmacodynamic model with an effect compartment model to describe the decrease in LVEF over time, related to trastuzumab exposure [42]. Recovery of the LVEF was implemented in the model. Additionally, the model incorporated the prior cumulative anthracycline dose as a covariate and found that this dose was an important determinant for the sensitivity to LVEF decline.

The relation between exposure and increase in BP and decrease of LVEF, as reported in both papers, are empirical models [40, 42]. It is, therefore, difficult to extrapolate these models directly between different drugs that might induce hypertension.

\section{QT interval prolongation}

Anti-cancer drugs, such as anthracyclines and tyrosine kinase inhibitors, can prolong the QT interval, which can lead to severe cardiac arrhythmias, such as torsade de pointes [38]. Concentration-QT modeling can provide important information on the relation between exposure and heart rate-corrected QT interval (QTc) [43, 44]. However, these models are mainly developed for anti-arrhythmic drugs and not for anti-cancer drugs. A recent publication investigated the effect of moxifloxacine, a compound that prolongs the QT interval, by developing a PK-PD model for translational purposes [43]. The time course of the QT interval is described by the following three components: the individual heart rate correction, the circadian rhythm, and the drug effect:

$Q T=\left(Q T_{0} \cdot \frac{R R}{R R_{r e f}}\right)^{\alpha}+A \cdot \cos \left(\frac{2 \pi}{24}(t-\varnothing)\right)+E_{\text {drug }}$ 
Table 2 Pharmacodynamic models describing continuous and categorical adverse effects

\begin{tabular}{|c|c|c|c|c|c|c|}
\hline Reference & Drug & & $\mathrm{AE}^{\mathrm{a}}$ & Observed variable & $\operatorname{Par}^{\mathrm{b}}$ & Drug effect \\
\hline \multicolumn{7}{|c|}{ Continuous adverse effects } \\
\hline van Hasselt (2011) & {$[42]$} & Trastuzumab & Cardiotoxicity & LVEF & 3 & $E_{\max }$ \\
\hline Keizer (2010) & {$[40]$} & Lenvatinib & Hypertension & $\mathrm{BP}$ & 3 & Linear \\
\hline Hansson (2013) & {$[41]$} & Sunitinib & Hypertension & $\mathrm{dBP}$ and $\mathrm{sBP}$ & 3 & Linear \\
\hline Marostica (2015) & [43] & Moxifloxacine & QT prolongation & QTc & 4 & Linear \\
\hline \multicolumn{7}{|c|}{ Categorical adverse effects } \\
\hline Keizer (2010) & {$[40]$} & Lenvatinib & Proteinuria & CTC & 6 & Linear \\
\hline Hénin (2008) & {$[48]$} & Capecitabine & HFS & CTC & 10 & $E_{\max }$ \\
\hline Hansson (2013) & {$[41]$} & Sunitinib & HFS and fatigue & CTC & 12 & $E_{\max }$ \\
\hline Suleiman (2015) & [49] & Erlotinib & Rash and diarrhea & CTC & 6 & Linear \\
\hline
\end{tabular}

$H F S$ hand-foot syndrome, $C T C$ NCI-CTC-AE, $L V E F$ left ventricular ejection fraction, $B P$ blood pressure, $d$ diastolic, $s$ systolic, $Q T c$ heart rate-corrected QT interval

${ }^{\text {a }}$ Adverse effect

${ }^{\mathrm{b}}$ Number of model parameters estimated in structural pharmacodynamic model (fixed effects excluding drug effect parameters)

where $\mathrm{QT}_{0}$ represents the $\mathrm{QT}$ interval at baseline, $\mathrm{RR}$ is the heart rate, and $\mathrm{RR} / \mathrm{RR}_{\text {ref }}$ is multiplied by $\mathrm{QT}_{0}$ to correct for individual heart rate. $A$ and $\varnothing$ represent the amplitude and the phase of the circadian rhythm, respectively, and ED represent the drug effect. Subsequently, the probability of QT prolongation above a critical threshold (e.g., $>10$ or $>20 \mathrm{~ms}$ ) can be derived. An effect compartment can be considered for modeling the ECG time course [45]. Similar model structures could be used to model QT prolongation induced by anti-cancer drugs.

\section{Ordered categorical adverse effects}

Typically, cancer therapy-related adverse effects are graded using the ordered NCI-CTC-AE scale, ranging from 0 to 5 . This range represents no adverse effects ( 0 ) to mild, moderate, severe, life-threatening adverse effects, and lastly death (5). Adverse effects such as vomiting, diarrhea, rash, fatigue, and hand-foot syndrome (HFS) are solely described by this ordered categorical scale. A conventional approach to describe the relation between exposure and the occurrence of a certain grade is by statistically comparing the incidence of grades between different dose groups. Early models for these type of adverse effects used ordered logistic regression or proportional odds models $[46,47]$. Both comparing the incidence of adverse effects and the ordered logistic regression approach have shortcomings. In these analyses, only the most severe grade of the adverse effect observed in a patient is used. By comparing incidences, the already categorized data is dichotomized, leading to substantial loss of information and ignoring the time course of the effect. Furthermore, the dependency of the previous observed grade in predicting the probability of the occurrence of the next grade is not taken into account. This problem can be addressed by implementing a Markov process in the model. First-order Markov models take into account the value of the preceding observation. The proportional odds model can be extended with a first-order Markov model [48]. In this way, the probability of transition between severity grades of adverse effects depends on the preceding grade. Typically, the logit transformation is used to constrain values of probabilities between 0 and 1 , similar to the logistic regression approach. This approach has been used for modeling different anti-cancer-induced graded adverse effects (Table 2).

HFS has been described by a proportional odds model with a Markov process to model the cumulative probabilities of getting a grade 0,1 , or $\geq 2$ for HFS related to accumulation of capecitabine [49]. HFS and fatigue in sunitinib-treated patients have been modeled using a first-order Markov model that was similar to the extension of the proportional odds model [41]. Vascular endothelial growth factor receptor 3 (VEGFR-3) was identified as biomarker, and its relative change over time was modeled as predictor of the occurrence and severity of fatigue and HFS.

Keizer et al. used a Markov transition model to describe proteinuria in patients treated with the VEGFR inhibitor lenvatinib [40]. Using a compartmental structure, each adverse event grade is represented with a compartment, which in turn is denoted with its own differential equation. The probability of experiencing a certain grade is represented by the corresponding compartment amount. The amounts in all compartments sum up to 1 at any time. At each observation, these amounts are re-set to a full probability for the observed state and 0 for all other states, and hence, a first-order Markov property is introduced. The rate constants for the movement of these amounts (i.e., probabilities) between compartments, 
which reflect the transitions between the different grades, are then estimated. As Markov models potentially allow transition between all states in the model, assumptions can be made to reduce the number of parameters to be estimated. For this reason, in the analysis of Keizer et al., only the transitions between neighboring grades were estimated. The following differential equations were used:

$$
\begin{aligned}
& \frac{d P\left(G r_{0}\right)}{d t}=k_{10} \cdot P(1)-k_{01} \cdot P(0) \\
& \frac{d P\left(G r_{1}\right)}{d t}=k_{01} \cdot P(0)+k_{21} \cdot P(2)-k_{10} \cdot P(1)-k_{12} \cdot P(1) \\
& \frac{d P\left(G r_{2}\right)}{d t}=k_{12} \cdot P(1)+k_{32} \cdot P(3)-k_{21} \cdot P(2)-k_{23} \cdot P(2) \\
& \frac{d P\left(G r_{3}\right)}{d t}=k_{23} \cdot P(2)-k_{32} \cdot P(3)
\end{aligned}
$$

Recently, a modeling and simulation framework for erlotinib-induced rash and diarrhea in patients with NSCLC was published [50]. The model structure was similar to the model used by Keizer et al., which was a continuous-time Markov model. A general structure of a Markov model, incorporating 5 grades (0-4), is depicted in Fig. 2.

The use of Markov processes is preferred over use of the proportional odds model for modeling ordered graded adverse effects. Markov models allow use of total longitudinal data on graded toxicities over time. Furthermore, these models take the preceding grade observed into account, which enables the precise characterization of the dynamics of toxicity. The Markov models currently published are empirical models. More mechanistic elements can easily be introduced in these models, for instance, using latent variables describing the underlying pharmacodynamic effects. However, this underlying mechanism is in most cases unknown. The major drawback of analyzing graded or categorical data is the fact that information is lost by using categories. In some cases, there is not a sufficient number of observations of severely graded adverse effects. Therefore, grades are sometimes merged together, leading to loss of already categorized information. However, if the clinical relevance between merged grades is not profound, this is an acceptable approach. Though, if available, the underlying observations might be better than the use of grades (e.g., blood pressure, instead of grades for hypertension).

\section{Application of adverse effect models}

Developed models for adverse effects have been applied to support decision-making regarding treatment optimization and clinical development. Ideally, PK-PD modeling frameworks are developed that integrate data on pharmacokinetics, adverse effects, and efficacy. An example of such a framework is available for sunitinib [41]. This paper did not only include modeling of ANC, fatigue, blood pressure, and HFS but also investigated if adverse effects were predictive for overall survival. Hypertension and neutropenia were found predictive for overall survival, functioning as biomarkers for treatment response.

Adverse effect models can additionally support decision-making regarding dose adjustments and dose individualizations, using simulation methods. The previously described modeling and simulation framework for erlotinib-induced rash and diarrhea investigated the safety of high-dose erlotinib pulses $(1600 \mathrm{mg} /$ week $+50 \mathrm{mg} /$ day remaining week days) proposed, compared to the standard dose $(150 \mathrm{mg} /$ day $)$ and different other dosing regimens [50]. Based on a simulation analysis using the framework developed, severe rash was predicted to occur in $20 \%$ of patients treated with the pulsed dosing regimen, compared to $12 \%$ in patients treated with the standard dosing regimen. In contrast with the common perception, radiotherapy was found to attenuate erlotinib-induced rash significantly, which advocates for using erlotinib and radiotherapy together. The framework also included a survival model, finding that experiencing rash at any grade was associated with improved clinical efficacy in terms of survival, albeit not significantly. Another example demonstrated that modeling can be helpful for determining individual dose adjustment of capecitabine to reduce severe grade HFS while maintaining efficacy [51]. The paper reports a clinical trial simulation in which the proportional odds Markov model was used on individual patient data [49]. Intolerable HFS (grade $\geq 2$ ) was predicted for the next treatment cycle, based on the previous cycle for each patient. Dose adjustments were made accordingly. Individualized dose adjustments using the Markov model were compared to using standard dose adjustments and found to reduce the duration of intolerable HFS by 10 days without loss of efficacy. Both modeling frameworks are examples of how a modeling approach can support dose adjustments and dose individualizations using predictive simulation methods.

Lastly, modeling and simulation of adverse effect models can optimize treatment and support clinical trial designs. The hypertension model, discussed in this review, has been used to optimize treatment with lenvatinib [52]. This paper investigated four strategies to clinically manage lenvatinib-induced hypertension to maximize both the number of patients on treatment and the average dose level during treatment, with use of simulations. An adverse effect-guided dose titration could potentially increase drug exposure without additional toxicity. Additionally, a design where anti-hypertensive treatment was followed by lenvatinib dose reduction proved to keep a large number of patients on treatment. This approach aimed at minimizing treatment cessation due to toxicity in order to improve 


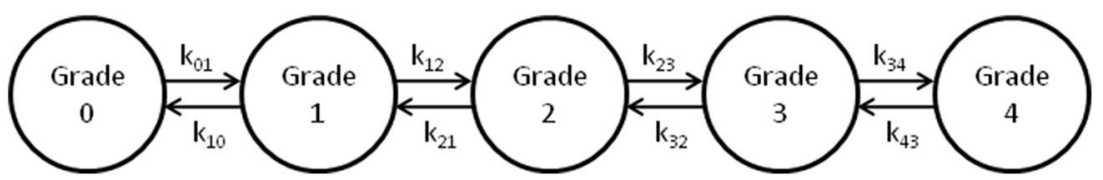

Fig 2 General structure of Markov model. Amount in compartment probability and $k_{x x}$ rate constants between probabilities [40, 50]

response to treatment. The intervention designs were supportive of development of a phase II clinical trial.

\section{Discussion}

This review reports several structural models for modeling adverse effects of anti-cancer drugs. Firstly, the best modeling approach depends on the type of data available and secondly, on whether or not prior knowledge of the underlying mechanism of the adverse effect is available. If the adverse effect is reported as continuous variable and prior knowledge on the mechanism behind the effect, the best approach is to develop a mechanism- or semi-mechanism-based model. Mechanistic models generally have a better predictive performance and potentially allow for extrapolation beyond the conditions on which the model was developed. The model by Friberg et al., published in 2002, proves to be the best starting point for modeling hematologic toxicity, with only few pharmacodynamic parameters to be estimated, and the mechanistic approach makes the model interchangeable between different anti-cancer drugs [17]. If no prior mechanistic knowledge is available or implementation in the model is impossible, data can be modeled using generic pharmacodynamic effect models. This more empirical approach can give insight in how an adverse effect evolves over time, if data is continuous. In some cases, the underlying continuous measurement is graded using the NCI-CTC-AE scale. For example, hypertension can be graded as such, however, the underlying continuous measurement, blood pressure, is needed to grade this toxicity. Therefore, blood pressure measurement itself can be used to develop PK-PD models. In conclusion, if an underlying continuous measurement is available, this longitudinal continuous data is preferred over ordered graded data, since it is less prone to loss of information. In subsequent simulation studies, the clinically well-accepted graded score can still be derived from the continuous data. Adverse effects like diarrhea, vomiting, and HFS are difficult to quantify and are described by ordered categorical grades. In this case, the best approach is to model the probabilities using a proportional odds model with a Markov process. The probability of a certain grade will then depend on the previously observed grade, which is true for almost all observed effects in oncology.

Modeling and simulation methods for analyzing adverse effects are preferred over the conventional comparison of adverse effect incidences between dosing groups. Quantitative models consider the variability between patients, allowing integration of patient characteristics that might be important in predicting the safety profile. Patient characteristics can alter systemic exposure to the drug and may lead to differences in onset, severity, and duration of adverse effects. Typically, physiological factors such as age, body size, gender, kidney function, and liver function can alter exposure, as well as pharmacogenetic factors and administration of other drugs [53]. Integration of these patient characteristics can be helpful in managing individual dose adaptations. In addition, proposed models can be used to model adverse effects driven by combination therapy, which is often applied in the oncology setting.

Established PK-PD models can predict different clinical scenarios. These simulations are particularly helpful in finding the optimal relationship between exposure and safety. Ideally, a PK-PD modeling framework is developed, that integrates data on exposure, efficacy, and toxicity, to assess the optimal balance between safety and efficacy [41, 54]. Modeling tumor growth as a biomarker for efficacy can be of added value in assessing this balance [55].

In conclusion, mathematical modeling of adverse effects can provide insight in how toxicities evolve over time and if or what patient-related factors can impact this time course. In addition, a modeling approach includes all available data, minimizing loss of information as is typically the case using more conventional methods of analyzing toxicity data. At last, modeling and simulation frameworks have been proven to support clinical trial designs, to optimize treatment, and to guide dose adjustments or dose individualizations. Therefore, modeling adverse effects proves to be a helpful tool for both improvement of clinical management and support of decisions regarding drug development.

Open Access This article is distributed under the terms of the Creative Commons Attribution 4.0 International License (http:// creativecommons.org/licenses/by/4.0/), which permits unrestricted use, distribution, and reproduction in any medium, provided you give appropriate credit to the original author(s) and the source, provide a link to the Creative Commons license, and indicate if changes were made.

\section{References}

1. Hryniuk W (1988) More is better. J Clin Oncol 6:1365-1367

2. Niraula S, Seruga B, Ocana A, et al. (2012) The price we pay for progress: a meta-analysis of harms of newly approved anticancer drugs. J Clin Oncol 30:3012-3019 
3. Tannock IF, Boyd NF, Deboer G, et al. (2012) Methotrexate, and fluorouracil chemotherapy for patients with metastatic breast cancer. J Clin Oncol 6:1377-1387

4. Sleijfer S, Wiemer E (2008) Dose selection in phase I studies: why we should always go for the top. J Clin Oncol 26:1576-1578

5. Hainsworth JD, Spigel DR, Burris HA, et al. (2010) Phase II trial of bevacizumab and everolimus in patients with advanced renal cell carcinoma. J Clin Oncol 28:2131-2136

6. Gray JE, Infante JR, Brail LH, et al. (2015) A first-in-human phase I dose-escalation, pharmacokinetic, and pharmacodynamic evaluation of intravenous LY2090314, a glycogen synthase kinase 3 inhibitor, administered in combination with pemetrexed and carboplatin. Investig New Drugs 33:1187-1196

7. Crawford J, Dale DC, Lyman GH (2004) Chemotherapy-induced neutropenia: risks, consequences, and new directions for its management. Cancer 100:228-237

8. Zhou H, Choi L, Lau H, et al. (2000) Population pharmacokinetics/ toxicodynamics (PK/TD) relationship of SAM486A in phase I studies in patients with advanced cancers. J Clin Pharmacol 40: 275-283

9. Jakobsen P, Bastholt L, Dalmark M, et al. (1991) A randomized study of epirubicin at four different dose levels in advanced breast cancer. Feasibility of myelotoxicity prediction through single blood-sample measurement. Cancer Chemother Pharmacol 28: 465-469

10. Minami H, Ando Y, Sakai S, Shimokata K (1995) Clinical and pharmacologic analysis of hyperfractionated daily oral etoposide. J Clin Oncol 13:191-199

11. Karlsson MO, Port RE, Ratain MJ, Sheiner LB (1995) A population model for the leukopenic effect of etoposide. Clin Pharmacol Ther 57:325-334

12. Karlsson MO, Molnar V, Bergh J, et al. (1998) A general model for time-dissociated pharmacokinetic-pharmacodynamic relationships exemplified by paclitaxel myelosuppression. Clin Pharmacol Ther 63:11-25

13. Haurie C, Dale DC, Mackey MC (1998) Cyclical neutropenia and other periodic hematological disorders: a review of mechanisms and mathematical models. Blood 92:2629-2640

14. Minami H, Sasaki Y, Saijo N, et al. (1998) Indirect-response model for the time course of leukopenia with anticancer drugs. Clin Pharmacol Ther 64:511-521

15. Friberg LE, Brindley CJ, Karlsson MO, Devlin AJ (2000) Models of schedule dependent haematological toxicity of 2'-deoxy-2'methylidenecytidine (DMDC). Eur J Clin Pharmacol 56:567-574

16. Zamboni WC, D'Argenio DZ, Stewart CF, et al. (2001) Pharmacodynamic model of topotecan-induced time course of neutropenia. Clin Cancer Res 7:2301-2308

17. Friberg LE (2002) Model of chemotherapy-induced myelosuppression with parameter consistency across drugs. J Clin Oncol 20:4713-4721

18. Panetta JC, Kirstein MN, Gajjar AJ, et al. (2003) A mechanistic mathematical model of temozolomide myelosuppression in children with high-grade gliomas. Math Biosci 186:29-41

19. Bulitta JB, Zhao P, Arnold RD, et al. (2009) Multiple-pool cell lifespan models for neutropenia to assess the population pharmacodynamics of unbound paclitaxel from two formulations in cancer patients. Cancer Chemother Pharmacol 63:1035-1048

20. Soto E, Staab A, Doege C, et al. (2011) Comparison of different semi-mechanistic models for chemotherapy-related neutropenia: application to BI 2536 a plk-1 inhibitor. Cancer Chemother Pharmacol 68:1517-1527

21. Sostelly A, Henin E, Chauvenet L, et al. (2013) Can we predict chemo-induced hematotoxicity in elderly patients treated with pegylated liposomal doxorubicin? Results of a population-based model derived from the DOGMES phase II trial of the GINECO. J Geriatr Oncol 4:34-45
22. Van Hasselt JGC, Gupta A, Hussein Z, et al. (2013) Population pharmacokinetic-pharmacodynamic analysis for eribulin mesilateassociated neutropenia. Br J Clin Pharmacol 76:412-424

23. Joerger M, Kraff S, Huitema ADR, et al. (2012) Evaluation of a pharmacology-driven dosing algorithm of 3-weekly paclitaxel using therapeutic drug monitoring: a pharmacokineticpharmacodynamic simulation study. Clin Pharmacokinet 51:607617

24. Trocóniz IF, Cendrós J-M, Soto E, et al. (2012) Population pharmacokinetic/pharmacodynamic modeling of drug-induced adverse effects of a novel homocamptothecin analog, elomotecan (BN80927), in a phase I dose finding study in patients with advanced solid tumors. Cancer Chemother Pharmacol 70:239-250

25. Hansson EK, Friberg LE (2012) The shape of the myelosuppression time profile is related to the probability of developing neutropenic fever in patients with docetaxel-induced grade IV neutropenia. Cancer Chemother Pharmacol 69:881-890

26. Kloft C, Wallin J, Henningsson A, et al. (2006) Population pharmacokinetic-pharmacodynamic model for neutropenia with patient subgroup identification: comparison across anticancer drugs. Clin Cancer Res 12:5481-5490

27. Friberg LE, Freijs A, Sandström M, Karlsson MO (2000) Semiphysiological model for the time course of leukocytes after varying schedules of 5-fluorouracil in rats. J Pharmacol Exp Ther 295:734-740

28. Soto E, Staab A, Freiwald M, et al. (2010) Prediction of neutropenia-related effects of a new combination therapy with the anticancer drugs BI 2536 (a Plk1 inhibitor) and pemetrexed. Clin Pharmacol Ther 88:660-667

29. Kaefer A, Yang J, Noertersheuser P, et al. (2014) Mechanism-based pharmacokinetic/pharmacodynamic meta-analysis of navitoclax (ABT-263) induced thrombocytopenia. Cancer Chemother Pharmacol 74:593-602

30. Quartino AL, Karlsson MO, Lindman H, Friberg LE (2014) Characterization of endogenous G-CSF and the inverse correlation to chemotherapy-induced neutropenia in patients with breast cancer using population modeling. Pharm Res 31:3390-3403

31. Chalret du Rieu Q, Fouliard S, White-Koning M, et al. (2014) Pharmacokinetic/pharmacodynamic modeling of abexinostatinduced thrombocytopenia across different patient populations: application for the determination of the maximum tolerated doses in both lymphoma and solid tumour patients. Investig New Drugs 32: 985-994

32. Hayes S, Mudd PN, Ouellet D, et al. (2013) Population PK/PD modeling of eltrombopag in subjects with advanced solid tumors with chemotherapy-induced thrombocytopenia. Cancer Chemother Pharmacol 71:1507-1520

33. Bender BC, Schaedeli-Stark F, Koch R, et al. (2012) A population pharmacokinetic/pharmacodynamic model of thrombocytopenia characterizing the effect of trastuzumab emtansine (T-DM1) on platelet counts in patients with HER2-positive metastatic breast cancer. Cancer Chemother Pharmacol 70:591-601

34. Slordal L, Spigset O (2006) Heart failure induced by non-cardiac drugs. Drug Saf 29:567-586

35. Lefrak EA, Pitha J, Rosenheim S, Gottlieb JA (1973) A clinicopathologic analysis of adriamycin cardiotoxicity. Cancer 32:302314

36. Keefe DL (2002) Trastuzumab-associated cardiotoxicity. Cancer 95:1592-1600

37. Chu W, Fyles A, Sellers EM, et al. (2007) Association between CYP3A4 genotype and risk of endometrial cancer following tamoxifen use. Carcinogenesis 28:2139-2142

38. Suter TM, Ewer MS (2013) Cancer drugs and the heart: importance and management. Eur Heart J 34:1102-1111

39. Senkus E, Jassem J (2011) Cardiovascular effects of systemic cancer treatment. Cancer Treat Rev 37:300-311 
40. Keizer RJ, Gupta A, Mac Gillavry MR, et al. (2010) A model of hypertension and proteinuria in cancer patients treated with the antiangiogenic drug E7080. J Pharmacokinet Pharmacodyn 37:347363

41. Hansson EK, Ma G, Amantea MA, et al. (2013) PKPD modeling of predictors for adverse effects and overall survival in sunitinibtreated patients with GIST. CPT Pharmacometrics Syst Pharmacol 2:e85

42. van Hasselt JGC, Boekhout AH, Beijnen JH, et al. (2011) Population pharmacokinetic-pharmacodynamic analysis of trastuzumab-associated cardiotoxicity. Clin Pharmacol Ther 90: 126-132

43. Marostica E, Van Ammel K (2015) Modelling of drug-induced QTinterval prolongation: estimation approaches and translational opportunities. J Pharmacokinet Pharmacodyn 42:659-679

44. Piotrovsky V (2005) Pharmacokinetic-pharmacodynamic modeling in the data analysis and interpretation of drug-induced QT/QTc prolongation. AAPS J 7:E609-E624

45. Holford N, Coates P, Guentert T, et al. (1981) The effect of quinidine and its metabolites on the electrocardiogram and systolic time intervals: concentration-effect relationships. Br J Clin Pharmacol 11:187-195

46. Mould D, Chapelsky M, Aluri J, et al. (2001) A population pharmacokinetic-pharmacodynamic and logistic regression analysis of lotrafiban in patients. Clin Pharmacol Ther 69:210-222

47. Xie R, Mathijssen RHJ, Sparreboom A, et al. (2002) Clinical pharmacokinetics of irinotecan and its metabolites in relation with diarrhea. Clin Pharmacol Ther 72:265-275
48. Zingmark PH, Kågedal M, Karlsson MO (2005) Modelling a spontaneously reported side effect by use of a Markov mixed-effects model. J Pharmacokinet Pharmacodyn 32:261-281

49. Hénin E, You B, Van Cutsem E, et al. (2009) A dynamic model of hand-and-foot syndrome in patients receiving capecitabine. Clin Pharmacol Ther 85:418-425

50. Suleiman AA, Frechen S, Scheffler M, et al. (2015) A modeling and simulation framework for adverse events in erlotinib-treated nonsmall-cell lung cancer patients. AAPS J 17:1483-1491

51. Paule I, Tod M, Hénin E, et al. (2012) Dose adaptation of capecitabine based on individual prediction of limiting toxicity grade: evaluation by clinical trial simulation. Cancer Chemother Pharmacol 69:447-455

52. Keizer RJ, Gupta A, Shumaker R, et al. (2012) Model-based treatment optimization of a novel VEGFR inhibitor. Br J Clin Pharmacol 74:315-326

53. Mathijssen RHJ, Sparreboom A, Verweij J (2014) Determining the optimal dose in the development of anticancer agents. Nat Rev Clin Oncol 11:272-281

54. Graham G, Gupta S, Aarons L (2002) Determination of an optimal dosage regimen using a Bayesian decision analysis of efficacy and adverse effect data. J Pharmacokinet Pharmacodyn 29:67-88

55. Ribba B, Holford NH, Magni P, et al. (2014) A review of mixedeffects models of tumor growth and effects of anticancer drug treatment used in population analysis. CPT Pharmacometrics Syst Pharmacol 3:e113 